\title{
Ten years of achievements in biological and medical sciences
}

\author{
Mingyao Liu* \& Tieliu Shi** \\ Shanghai Key Laboratory of Regulatory Biology, Institute of Biomedical Sciences and School of Life Sciences, East China Normal University, \\ Shanghai 200241, China \\ Received December 26, 2016
Citation: Liu, M., and Shi, T. (2017). Ten years of achievements in biological and medical sciences. Sci China Life Sci 60, 111-115. doi: 10.1007/s11427-017-9003-3

This special issue commemorates the 10th anniversary of Institute of Biomedical Sciences (IBMS), East China Normal University (ECNU). IBMS was established in 2007 by a group of oversea scientists from Harvard Medical School, Yale University, the Texas A\&M University, Baylor College of Medicine, the University of Texas MD Anderson Cancer Center, the University of California and the Scripps Research Institute. These scientists were brought together on the initiative of Dr. Mingyao Liu, who was then appointed as the director of IBMS and dean of School of Life Sciences, ECNU. The institute is located on the new ECNU campus in Minhang district of Shanghai. IBMS has a mission to promote and advance biological and medical sciences in all their forms through research, education, communication and collaboration. The main purpose of IBMS is to conduct forefront basic and applied research in cell signaling transduction and regulatory biology. Its research focus includes gene transcriptional regulation, protein modification, stem cell and developmental regulation, and innovative drug discovery. In 2011, the institute was awarded with the title of Shanghai Key Laboratory of Regulatory Biology, with the direct support from Shanghai Municipal Science and Technology Commission. In 2013, it won the most outstanding laboratory in Shanghai.

As one of the centers for education and research in China, IBMS tries to lead the way in research and application of medical sciences. From the first founding with a few scientists to a big team composed of outstanding young and

*Corresponding authors (email: myliu@bio.ecnu.edu.cn)

**Corresponding authors (email: tlshi@bio.ecnu.edu.cn) senior scientists in multifaceted research areas today, the growth of the IBMS is quite promising. IBMS currently has 16 independent research groups and more than 60 full-time faculties and staffs. IBMS has totally received more than 150 grants from the Ministry of Science and Technology, the Ministry of Education, and the National Natural Science Foundation of China, as well as Shanghai Municipal Science and Technology Commissions.. During the past 10 years, members of IBMS have published more than 300 articles in peer-reviewed and high-impact journals, including Nature Biotechnology, Nature Medicine, Immunity, Nature Protocols, Cell Metabolism, Nature Communications, Molecular Cell, Proceedings of the National Academy of Sciences of the United States of America, Cell Research and Cancer Research, etc. Based on the great achievements in medical sciences, faculty of IBMS was awarded the innovative team of the Ministry of Education in 2012, and IBMS received continuous financial support from the Ministry of Education in 2015. Importantly, a special educational system and a set of courses have been developed in IBMS to help students to develop their skills, increase their knowledge, and advance their careers in molecular biology, drug development, translational research, and related fields. Numerous talented and outstanding students have graduated from IBMS as educators, researchers as well as biological company founders in China and worldwide.

In this Science China Life Sciences special issue, ten articles contributed by seven different research groups led by Jiemin Wong, Dali Li, Yuping Lai, Yuan Wang, Tieliu Shi, Min Qian and Mingyao Liu, cover a wide spectrum of medical sciences. Researches in Dr. Jiemin Wong's group have 
been focused on epigenetic regulation and transcriptional regulation. His group has made significant contributions to the identification and characterization of the corepressor complexes of nuclear hormone receptors. In this special issue, Dr. Jiemin Wong and his colleagues identify a group of acidic-domain containing proteins, which are a novel histone-binding proteins family. This protein family differentially reads the status of $\mathrm{H} 3 \mathrm{~K} 4$ methylation (Wu et al., 2017). Dr. Dali Li's group pursues to apply genome editing technology in the generation of animal models and therapy of genetic diseases. By using transcription activator-like effector nucleases technology, they generate Leptin receptor knockout rats on the Sprague Dawley genetic background (Chen et al., 2017). Dr. Yuping Lai is interested in the exploration of underlying mechanisms in wound healing and psoriasis. In this issue, her study shows that activation of Toll-like receptor 3 signaling induces keratinocyte differentiation by upregulation of S100A7 (psoriasin) (Lei et al., 2017). Dr. Yuan Wang's research has been focused on the genetic programs that govern hematopoietic development and germ cell differentiation from embryonic stem cells. By utilizing an in vitro differentiation system of mouse embryonic stem cells, Dr. Yuan Wang and her co-workers identify several microRNAs with unknown functions that are highly enriched in embryonic hematopoietic cells (Gong et al, 2017). Dr. Tieliu Shi's research focuses are on data analyses with integration of multi-level data (including NGS sequencing data, Proteomics data and clinical data, etc.) for disease genes, disease mechanisms and biomarker discoveries and prediction of gene regulatory network and protein-protein interaction. His group systematically compares human orthologs with mouse orthologs in terms of alternative splicing patterns and expression profiles (Chen et al, 2017), and annotates genomes using RNA-seq data (Chen et al., 2017). Dr. Min Qian and her team's research focuses on the molecular mechanisms of macrophage mediated innate immune responses in inflammation, cancer and infectious diseases. They reveal that Presenilin 2 (PS2) deficiency facilitates $A \beta$-induced neuroinflammation and injury by upregulating $\mathrm{P} 2 \mathrm{X} 7$ expression, which provide a potential new mechanism for Alzheimer's disease (Qin et al, 2017). The rest three articles of this special issue are contributed by the team led by Dr. Mingyao Liu. His team has been focused on basic research to understand physiological functions of G-protein coupled receptors, and translational research to discover innovative drugs by targeting critical cell signaling pathways in cancers. Dr. Mingyao Liu and his colleagues summarize molecular targets of human gastric cancer based on collaborative sequencing data (Guo et al, 2017), discover a novel angiogenesis inhibitor (Liu et al., 2017) and set up pharmacokinetic platform to evaluate compounds' activity on metabolic enzymes (Lu et al, 2017). Besides the above research groups, there are other groups in IBMS that focus on structure biology, immunology, synthetic biology and functional proteomics.

IBMS has been working on building leadership in biomedical research and sciences during the past 10 years. We hope that IBMS will create more significant knowledge and apply more science and discoveries to further education, and this goal will be realized through collaborative research across disciplines and specialties locally and globally.

Compliance and ethics The author(s) declare that they have no conflict of interest.

Chen, G., Chen, J., Yang, J., Chen, L., Qu, X., Shi, C., Ning, B., Shi, L., Tong, W., Zhao, Y., Zhang, M., and Shi, T. (2017). Significant variations in alternative splicing patterns and expression profiles between human-mouse orthologs in early embryos. Sci China Life Sci 60, 178-188.

Chen, G., Shi, T., and Shi, L. (2017). Characterizing and annotating the genome using RNA-seq data. Sci China Life Sci 60, 116-125.

Chen, Y., Lu, W., Gao, N., Long, Y., Shao, Y., Liu, M., Chen, H., Ye, S., Ma, X., Liu, M., and Li, D. (2017). Generation of obese rat model by transcription activator-like effector nucleases targeting the leptin receptor gene. Sci China Life Sci 60, 152-157.

Gong, X., Chao, R., Wang, P., Huang, X., Zhang, J., Zhu, X., Zhang, Y., Yang, X., Hou, C., Ji, X., Shi, T., and Wang, Y. (2017). Interplay of transcription factors and microRNAs during embryonic hematopoiesis. Sci China Life Sci 60, 168-177.

Guo, J., Yu, W., Su, H., and Pang, X. (2017). Genomic landscape of gastric cancer: molecular classification and potential targets. Sci China Life Sci 60, 126-137.

Lei, H., Wang, Y., Zhang, T., Chang, L., Wu, Y., and Lai, Y. (2017). TLR3 activation induces $\mathrm{S} 100 \mathrm{~A} 7$ to regulate keratinocyte differentiation after skin injury. Sci China Life Sci 60, 158-167.

Liu, Y., He, Y., Yang, F., Cong, X., Wang, J., Peng, S., Gao, D., Wang, W., Lan, L., Ying, X., Liu, M., Chen, Y., and Yi, Z. (2017). A novel synthetic small molecule YF-452 inhibits tumor growth through antiangiogenesis by suppressing VEGF receptor 2 signaling. Sci China Life Sci 60, 202-214.

Lu, J., Ding, T., Qin, X., Liu, M., and Wang, X. (2017). In vitro and in vivo evaluation of cucurbitacin $\mathrm{E}$ on rat hepatic CYP2C11 expression and activity using LC-MS/MS. Sci China Life Sci 60, 215-224.

Qin, J., Zhang, X., Wang, Z., Li, J., Zhang, Z., Ren, H., Qian, M., and Du, B. (2017). Presenilin 2 deficiency facilitates A $\beta$-induced neuroinflammation and injury by upregulating P2X7 expression. Sci China Life Sci 60, 189-201.

Wu, M., Wei, W., Chen, J., Cong, R., Shi, T., Li, J., Wong, J., and Du, J.X. (2017). Acidic domains differentially read histone H3 lysine 4 methylation status and are widely present in chromatin-associated proteins. Sci China Life Sci 60, 138-151. 


\section{Biographical Sketch}

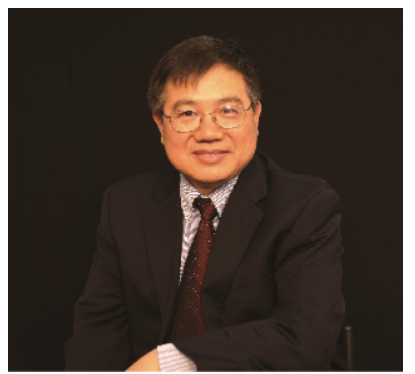

Dr. Mingyao Liu received his Ph.D. Degree in Cell Biology from the University of Maryland, College Park in 1992. From 1993 to 1998, he did his postdoctoral trainings in Johns Hopkins University School of Medicine and California Institute of Technology (Caltech) with Prof. King-Wai Yau and Melvin I Simon. In 1999, he was recruited to the Institute of Biosciences and Technology, Texas A\&M University as an assistant professor, then promoted to associate professor in 2003 and full professor with tenure in 2007. In 2007, Dr. Liu was recruited as the director of the Institute of Biomedical Sciences, then appointed as the dean of School of Life Sciences and the director of Shanghai Key Laboratory of Regulatory Biology, East China Normal University, China in 2012. Together with his colleagues, Dr. Liu established a laboratory for cell signaling and innovative drug discovery. In 2008, he was selected as the Distinguished Endowed Professor by "The Recruitment Program of Global Experts". In 2014, he was awarded Shanghai Magnolia Prize and First Prize of National Scientific and Technological Progress Award.

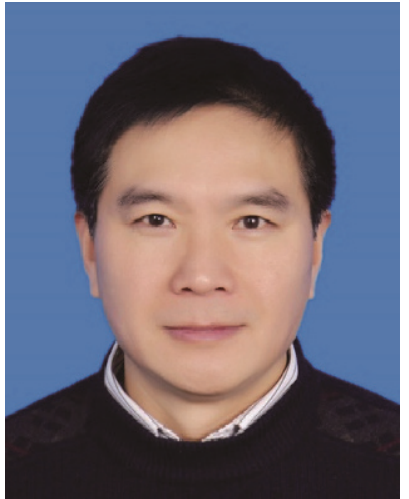

Dr. Tieliu Shi received his Master degree in computer science in 1999 and Ph.D. degree in molecular biology in 2000 from the University of Louisville. After graduate school, he pursued bioinformatics research and joined the Bioinformatics Center, Shanghai Institute of Biological Sciences, Chinese Academy of Sciences between 2002 and 2008. He moved to East China Normal University by the end of 2008 and served as full professor. He has various research interests, including (1) Disease gene, disease mechanism and biomarker discoveries based on multi-level data integration of omics and clinical information; (2) Methodology developments and applications in the high through-put data (NGS data, proteomic data, etc.); (3) Gene regulatory network prediction and protein-protein interaction network prediction; (4) Drug target, drug efficacy and adverse prediction, including Traditional Chinese Medicine.

Dr. Jiemin Wong received his bachelor degree in Biology in 1984 from Wuhan University, M.S. degree in Cell Biology in 1987 from Shanghai Institute of Cell Biology and Ph.D. degree in Molecular Genetics and Microbiology in 1994 from University of Vermont. He had his postdoc training in NIH during 1994-1997. He joined the Dept of Molecular and Cellular Biology, Baylor College of Medicine as an Assistant Professor in 1997 and promoted to tenured Associate Professor in 2003. Dr. Wong joined the School of Life Sciences and the Institute of Biomedical Sciences in East China Normal University in 2007 and since then has served as Professor and Associate Director for the Institute of Biomedical Sciences. Dr. Wong's laboratory has long been interested in epigenetic regulation and transcriptional regulation by nuclear hormone receptors (NRs). His group has made contribution to the identification and characterization of the corepressor complexes of nuclear hormone receptors, histone demethylases and identification and characterization of histone code effectors. He has published more than 100 peer-reviewed papers. His current research focuses on epigenetic regulation including DNA methylation and histone modifications.

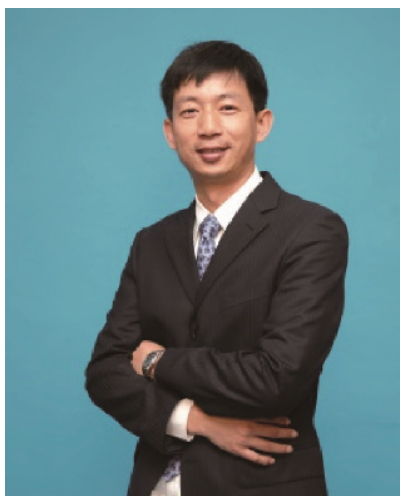

Dr. Dali Li completed his B.S. degree in 2001 and obtained his Ph.D. degree in Genetics from Hunan Normal University in 2007. As a visiting scholar, he joined Mingyao Liu's lab in Texas A\&M University Health Science Center in 2004-2007. He became a lecture in School of Life Sciences, East China Normal University in 2007 and was promoted to Professor in 2014. He has set up and directs the transgenic core facility in ECNU. He is interested in applying and developing of novel genome modification technologies for disease model generation and in vivo gene therapy. 

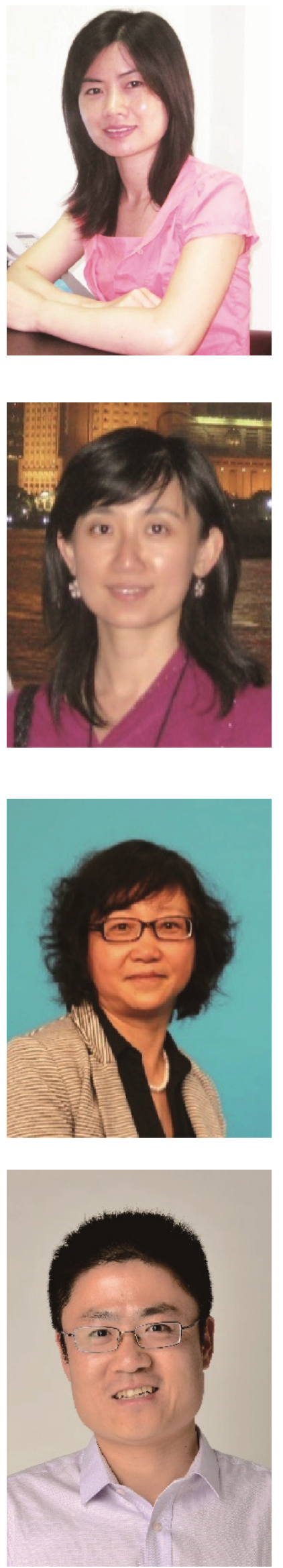

Dr. Yuping Lai received her Ph.D. degree from East China Normal University in 2006. After her graduate school, which included an NIH/NIAID predoctoral fellowship, Yuping moved to Dr. Gallo's lab in University of California-San Diego and began to investigate the relationships between microbes and their hosts, especially how Staphylococcus epidermidis influences skin immune responses. In 2010 Yuping moved back to China and started her own laboratory in East China Normal University. Now her research interests are focused on understanding innate immune responses involved in wound infection, inflammation and wound healing, especially including: (1) investigating the functions of REG3A in wound healing, wound inflammation and the pathogenesis of psoriasis; (2) exploring the mechanisms by which IL-1 family cytokines induce REG3A in keratinocytes; (3) understanding how hyperglycemia facilitates Staphylococcus aureus skin infection in diabetes.

Dr. Yuan Wang obtained her Ph.D. degree from Boston University in Molecular and Cell Biology and Biochemistry in 2002. She completed her post-doc training at Harvard Medical School and NIEHS afterwards. Dr. Wang joined East China Normal University as a professor in 2009. The main research focus of Dr. Wang's lab is to dissect the genetic programs that govern germ cell and blood differentiation using both in vitro differentiation system of PSCs and gene modified mouse models. She is also interested in molecular mechanism that regulates self-renewal of PSCs.

Dr. Min Qian completed her B.S. degree at East China Normal University in 1983. After that, she earned her Master degree at East China Normal University in 1986. Then, she began her assistant professor position at Suzhou University of Science and Technology from 1986 to 1988. She obtained her PhD degree at East China Normal University in 1991 and then served as an assistant professor at East China Normal University, and has been a full professor of immunology since 2002. She is interested in exploring specific human antibody to different drug targets by using phage display technology. Meanwhile, she is also involved in understanding the regulation of innate immune responses in inflammation, cancer and infectious diseases.

Dr. Bing Du completed his B.S. degree in biochemistry from Anhui University in 2001. After that, he earned his Ph.D. degree in biochemistry and molecular biology at East China Normal University in 2006. Since then, he began his assistant professor position at East China Normal University, and is now an associate professor of immunology from 2010. In this period, he joined Dr. Mingyao Liu's laboratory as a visiting scholar for more than 1 year at Texas A\&M Institute of Biosciences and Technology in Houston. His research interests are in understanding the molecular mechanisms of macrophage mediated innate immune responses in inflammation, cancer and infectious diseases. 


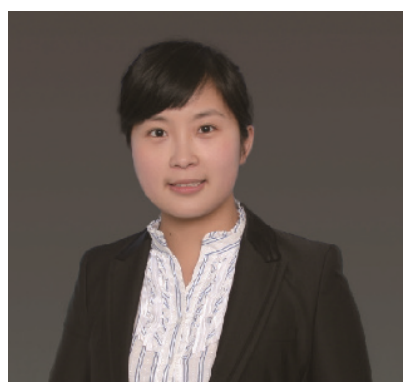

Dr. Xiufeng Pang received her Ph.D. degree in 2009 from the joint program between East China Normal University (ECNU), Shanghai, China, and Texas A\&M University Health Science Center, Texas, US. Upon her completion of Ph.D. training, she joined School of Life Sciences, ECNU as a research associate and studies in the fields of anticancer drug discovery and molecular pharmacology. In 2012, Dr. Pang became an associate professor and the main leader of anticancer drug screening section, Shanghai key laboratory of regulatory biology. Dr. Pang published more than 30 articles on high-impact biological and pharmacological journals, such as Nature Communications, Cancer Research, Molecular Cancer Therapeutics, Carcinogenesis, and Journal of Medicinal Chemistry, etc. She got several grants from National Natural Science Foundation, Shanghai Municipal Education Commission and Science and Technology Commission of Shanghai Municipality. Dr. Pang is the recipient of Chenguang Research Award from Shanghai Municipal Education Commission, State Scholarship from China Scholarship Council and Biovision.Nxt. Award from International Bioscience Forum. Her major scientific focus includes novel anticancer drug development, clinical anticancer drug resistance and combined therapy.

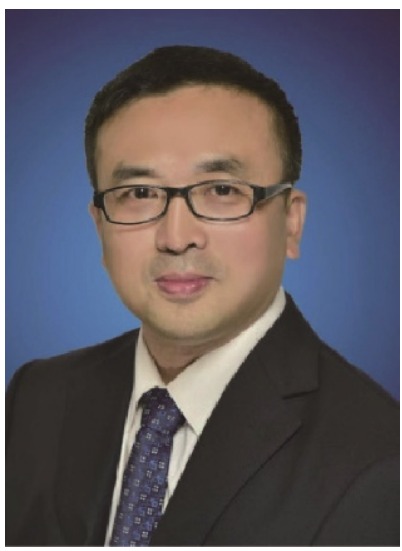

Dr. Zhengfang $\mathrm{Yi}$ is a professor and an investigator in the Biomedical research Institute at East China Normal University, Shanghai, China. He received his master's degree from Hunan Normal University (Hunan, China) in 2004. He obtained his Ph.D. from East China Normal University and the Texas A\&M University (USA). He was promoted to Associate Professor in 2010 and to Professor in 2015. He is interested in the development of new drugs for tumor (prostate cancer, breast cancer, leukemia, colon cancer, liver cancer) as well as new medical devices.

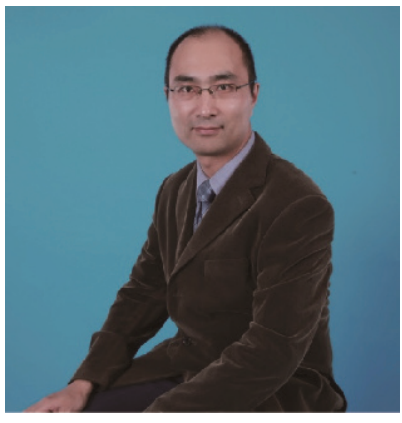

Dr. Xin Wang received his Ph.D. degree in pharmacology from the Chinese University of Hong Kong in 2007, and completed his postdoctoral training in the University of Texas Medical Branch in 2011. He has been working in the East China Normal University as associate professor since 2011. Dr. Wang has the honor to win the Youth Pharmacologist Award of Chinese Pharmacological Society in 2016. His research fields include pharmacokinetics and drug metabolism, especially drug-drug interactions, and novel models for ADME, such as 3D organoids of transporter protein, CYP knockout rat and humanized rat model. Currently, Dr. Wang is hosting or participating in many projects supported by the Science and Technology Commission of Shanghai Municipality and the National Natural Science Foundation of China. Dr. Wang has published more than 40 SCI papers in international peer-reviewed journals, such as Nature Communications, Biochemical Pharmacology, and Pharmacological Research, etc. Moreover, he also edited one pharmacological book, and contributed four book chapters. Meanwhile, he is dedicated to social activities and serves as the editorial board member and ad-hoc reviewer of 27 SCI journals, such as Biochemical Pharmacology, Journal of Pharmaceutical and Biomedical Analysis, and Current Drug Metabolism, etc. 